\section{Kidney Blood Pressure Research}

\title{
Prognostic Factors of Renal Involvement in Systemic Sclerosis
}

\author{
Edoardo Rosato ${ }^{a} \quad$ Antonietta Gigante $^{a}$ Biagio Barbano ${ }^{a}$ \\ Maria Ludovica Gasperini ${ }^{a}$ Rosario Ciancib Maurizio Muscaritolia \\ aDepartment of Clinical Medicine-Scleroderma Unit, Sapienza University of Rome, Rome, ${ }^{\mathrm{b}}$ Department \\ of Clinical Medicine, Sapienza University of Rome, Rome, Italy
}

\section{Key Words}

Systemic sclerosis - Scleroderma renal crisis - Intrarenal arterial stiffness - Resistive index • Color Doppler ultrasound

\begin{abstract}
Background/Aims: Renal involvement is common in systemic sclerosis (SSc), including asymptomatic reduction of glomerular filtration rate (GFR), increased renal resistance indices, scleroderma renal crisis (SRC) and ANCA-associated vasculitis. The aim was to evaluate type and evolution of renal involvement for a period of five years. Methods: $121 \mathrm{SSc}$ patients (100 F, $21 \mathrm{M}$ ) with mean age of $54.9 \pm 13.8$, disease duration of $9 \pm 6$ years, of which 62 had a diffused form and 59 limited form were enrolled. All patients were screened annually for renal function by laboratory examination, ultrasound and color Doppler ultrasound of renal arteries. Results: Over the five-year observation period, 6 SRC (3 M, 3 F) occurred, four of which required dialysis. One patient developed ANCA-related proliferative glomerulonephritis and the other one acute tubular necrosis. The remaining 113 patients had a preserved renal function (serum creatinine $0.75 \pm 0.24 \mathrm{mg} / \mathrm{dl}$, GFR $93.8 \pm 20 \mathrm{ml} / \mathrm{min}, 24 \mathrm{~h}$ proteinuria $0.20 \pm 0.15 \mathrm{~g}$ ). Doppler indices of intrarenal arterial stiffness increased with progression of capillaroscopic damage and with presence of digital ulcers. A negative correlation was observed between estimated GFR and pulsatile index $(p<0,05, r=-0.198)$, resistive index $(p<0,01, r=0.267), S / D$ ratio $(p<0,01$, $r=-0.237)$. Conclusion: In SSc patients, renal function was normal for 4.1 years despite the presence of increased intrarenal arterial stiffness. SRC was observed in $4.9 \%$ of SSc patients. In SSc patients, a periodic follow-up based on clinical and laboratory evaluation, colorDoppler ultrasound and, in some cases, renal biopsy is required to evaluate renal involvement.
\end{abstract}




\section{Kidney Blood Pressure Research}

\section{Introduction}

Systemic sclerosis (SSc) is an autoimmune disease characterized by collagen deposition and fibrosis of skin and internal organs [1]. The hallmarks of SSc are related to endothelial damage, production of autoantibodies and fibroblast dysfunction [2].

Consistently, renal involvement is frequently described in SSc, including high intrarenal arterial stiffness [3], isolated reduced glomerular filtration rate (GFR), reduced renal functional reserve, microalbuminuria and proteinuria, penicillamine-associated renal disease, antiphospholipid-associated nephropathy, Myeloperoxidase-Antineutrophil Cytoplasmic Antibody (MPO-ANCA) associated glomerulonephritis and vasculitis, to scleroderma renal crisis (SRC) and normotensive SRC [4].

Notably, manifestations of renal disease are observed clinically in up to $50 \%$ of SSc patients [5], even if autopsy studies revealed histological evidence of renal impairment in up to $80 \%$ of these patients [6].

In the Australia and New Zealand Dialysis and Transplant (ANZDATA) registry, $0.3 \%$ of the population (127 patients) developed end stage renal disease (ESRD) secondary to SSc. Systemic sclerosis is an uncommon cause of ESRD, which is associated with increased risks of both dialysis-independent renal function recovery and patient mortality compared with other causes of ESRD [7].

Steen et al, in 2005, studied the frequency and severity of kidney abnormalities in diffuse cutaneous SSc patients and they found SRC in 129/675 (19.5\%) patients and reduced renal function or proteinuria secondary to toxicity from D-penicillamine in 173 patients $(26 \%)$.

The aim of the present study was to prospectively evaluate prevalence, type and evolution of resistive index (RI) in patients with limited (lcSSc) and diffuse cutaneous (dcSSc) Systemic Sclerosis admitted to a Scleroderma Unit over a period of five years.

\section{Materials and Methods}

121 patients who met the American College of Rheumatology/European League Against Rheumatism Collaborative Initiative criteria for SSc admitted to the Scleroderma Unit of the Department of Clinical Medicine, Sapienza University of Rome were enrolled in the study [8]. Fifty-nine (48.8\%) patients had lcSSc and sixtytwo (51.2\%) presented dcSSc as defined by LeRoy et al. [9]. Table 1 shows patients' epidemiological and clinical features. No patients with diabetes were enrolled. Patients mean follow-up was $4.1 \pm 0.9$ years, including laboratory tests and renal ultrasound indices. All patients were in therapy with nifedipine 20-30 mg day for Raynaud's phenomenon. Seventy-five patients were treated with iloprost $(0,5-2 \mathrm{ng} / \mathrm{kg} / \mathrm{min}$ for 8 hours for three days monthly) for treatment of digital ulcers (DUs) and Raynaud's phenomenon. Fifty SSc patients received bosentan therapy ( $250 \mathrm{mg} /$ day) for DUs prevention. The subjects' written consent was obtained according to the Declaration of Helsinki and the study was approved by ethics committee of Sapienza University.
Table 1. Characteristics of the patients studied. Diffuse cutaneous SSc (dcSSc), limited cutaneous SSc (lcSSc), Disease Activity Index (DAI) and disease severity scale (DSS)

\begin{tabular}{lc}
\hline Characteristics & Value \\
\hline Sex (female/male) & $100 / 21$ \\
Age (years) & $54.9 \pm 13.8$ \\
Disease duration (years) & $9 \pm 6$ \\
DAI & $2.5 \pm 2.1$ \\
DSS & $4.9 \pm 3.1$ \\
dcSSc/lcSSc & $62 / 59$ \\
Digital ulcers history & $64(52.9)$ \\
& \\
SSc-specific autoantibodies & $\mathrm{n}$ \\
Anti-topoisomerase I & 64 \\
Anti-centromere & 48 \\
RNA polymerase III & 3 \\
None & 6 \\
& \\
Capillaroscopic pattern & $\mathrm{n}(\%)$ \\
Early & $29(24)$ \\
Active & $46(38)$ \\
Late & $46(38)$ \\
\hline
\end{tabular}




\section{Kidney Blood Pressure Research}

Rosato et al.: Kidney and Systemic Sclerosis

\section{Clinical assessment}

In all patients nailfold videocapillaroscopy (NVC) was performed (Videocapillaroscope Pinnacle Studio Version 8, equipped with a 500× optical probe). According to Cutolo et al., patterns identified within the "SSc pattern" include: early, active and late [10]. Modified Rodnan total skin score (mRSS) used to assess skin involvement in SSc patients [11]. Disease activity in SSc was measured using Disease Activity Index (DAI) [12] and disease severity was measured by Medsger Disease Severity Scale (DSS) [13]. Arterial hypertension was diagnosed according to ESH/ESC Guidelines for the management of arterial hypertension. Hypertension was defined as values $>140 \mathrm{mmHg}$ SBP and/or $>90 \mathrm{mmHg}$ DBP [14].

\section{Laboratory parameters}

Laboratory parameters included serum creatinine $(\mathrm{sCr}$ ) (normal range: $0.5-0.9 \mathrm{mg} / \mathrm{dl}$ ), blood urea nitrogen (normal range: $10.20-49.80 \mathrm{mg} / \mathrm{dl}$ ), urinalysis and $24 \mathrm{~h}$ proteinuria. GFR was calculated with CKDEPI equation [15].

\section{Renal ultrasound}

Color Doppler Ultrasound of kidney (CDU) evaluated renal arteries to detect morphologic abnormalities or renal artery stenosis (convex 3.5-MHz probe, Toshiba Aplio Ultrasound System SSA-790, Tokyo, Japan).

A single blinded physician performed all the ultrasound examinations. The average of three measurements for each Doppler parameter was calculated in both kidneys at the level of arcuate and interlobar arteries. Peak systolic velocity (PSV), end diastolic velocity (EDV), resistive index (RI), pulsatile index (PI), and systolic/diastolic ratio (S/D) were measured.

RI was calculated as it follows: (peak systolic frequency shift - minimum diastolic frequency shift)/peak systolic frequency shift. PI was calculated as it follows: (peak systolic frequency shift)/minimum diastolic frequency shift)/mean frequency shift. We considered as pathologic a value of RI>0.7 [16]. Calcium channel blockers and bosentan therapy was discontinued 72 hours before the CDU examination. Patients receiving iloprost underwent Doppler examination the day before the next infusion.

\section{Statistical analysis}

The results were expressed as mean \pm SD or median and range as appropriate. Commercially software (SPSS version 24.0) was used for statistical analysis. The coefficient of skewness and the coefficient of kurtosis were used to evaluate the normal distribution of data. Multiple regression analysis was applied to evaluate the relationship between age and Doppler indices of intrarenal stiffness and others quantitative variables. Pearson or Spearman correlation coefficient ( $r$ ) was used to test for bivariate analysis. Group comparisons were made by Student's unpaired 2-tailed t-test or the Kruskal-Wallis test, as appropriate. P-values $<0,05$ were considered significant.

\section{Results}

Table 2 shows mean values of Doppler indices of intrarenal stiffness, sCr, CKD-EPI, 24h proteinuria and intimal media thickness (IMT) in SSc patients at baseline (T0) and after follow-up of $4.1 \pm 0.9$ years (T1). No significant differences in these parameters were observed between T0 and T1. Arterial hypertension was seen in 21 (17.4\%) of these patients and their mean peak blood pressure at diagnosis was 161/98 $\mathrm{mmHg}$. New hypertension was diagnosed only in 3 patients (2, 5\%). Fifteen were in treatment with nifedipine $30 \mathrm{mg}$ day with good control of blood pressure. Six patients were in treatment with nifedipine $30 \mathrm{mg}$ day plus losartan $50 \mathrm{mg}$.

Scleroderma Renal Crisis occurred in six (4.9\%) patients (3 males, 3 females) of which five had dcSSc and one lcSSc. Three patients had anti-topoisomerase antibody, two had anti RNA-polymerase III antibody and one had an anti-centromere antibody. Two of them received $10 \mathrm{mg}$ of prednisone in the previous six and eight months. Four of them required dialysis and two of them died after nine and twelve months of SRC onset; one patient remained in peritoneal dialysis and recovery of dialysis-independent renal function occurred in one 


\section{Kidney Blood Pressure Research}

patients after 24 months of SRC onset. The other two patients had a favorable prognosis and resolved the SRC within one month, following high doses of Ramipril.

MPO-ANCA associated glomerulonephritis was diagnosed in one patient who died 3 months later, due to infectious complications; acute tubular necrosis was diagnosed in another patient who required dialysis only for 2 months and she remains in stage 3 chronic kidney disease. These patients were excluded from follow-up.

Table 3 shows the median values of Doppler indices of intrarenal stiffness, sCr, CKDEPI, proteinuria $24 \mathrm{~h}$ in six SSc patients with SRC at baseline (T0), during SRC (T1) and median follow-up of 60 (9-60) months (T2). The RI showed a significant increase during SRC and it return at normal value during the follow-up. At T1 and T2 $\mathrm{sCr}$ significantly $(\mathrm{p}<0.05)$ increased, conversely CKD-EPI significantly $(\mathrm{p}<0.05)$ reduced.

The remaining 113 patients had a preserved renal function $(\mathrm{sCr} 0.75 \pm 0.24 \mathrm{mg} / \mathrm{dl}$, CKDEPI $93.8 \pm 20 \mathrm{ml} / \mathrm{min}, 24 \mathrm{~h}$ proteinuria $0.20 \pm 0.15 \mathrm{~g}$ ). The Doppler indices of intrarenal arterial stiffness were: PI $1.43 \pm 0.28$, RI $0.70 \pm 0.05$, and S/D $3.50 \pm 0.76$. A negative correlation was observed between CKD-EPI and PI ( $p<0,05, r=-0.198)$, RI ( $p<0,01, r=0.267), S / D$ ratio $(\mathrm{p}<0,01, r=-0.237)$. At baseline, the mean value of PI, RI and SD significantly increased with progression of capillaroscopy damage (Table 4). Any difference of sCr, CKD-EPI and IMT was observed in three capillaroscopic groups (Table 4). At baseline the mean values of PI, RI and S/D were significantly higher in SSc patients with DUs than in SSc without DUs. Any difference of sCr, CKD-EPI and IMT was observed in SSc patients with or without DUs (Table $5)$.

In multiple regression analysis, age showed a linear positive correlation with $\mathrm{sCr}$ $(\mathrm{p}<0,0001, \mathrm{r}=0,16)$ and IMT $(\mathrm{p}<0,0001, \mathrm{r}=0$, $63)$ and a linear negative correlation with CKDEPI $(p<0,0001, r=-0,58)$. Age did correlate with Doppler indices of intrarenal stiffness.

In SSc patients with arterial hypertension RI ( $p<0,01,0,73 \pm 0,06$ vs $0,70 \pm 0,05)$ and S/D ( $<<0,01,3,93 \pm 0,93$ vs $3,41 \pm 0,68)$ were significantly higher than in patients without arterial hypertension. Any significant difference (p>0, 05) of PI $(1,50 \pm 0,27$ vs 1 , $42 \pm 0,28), \operatorname{sCr}(0,75 \pm 0,26$ vs $0,73 \pm 0,06)$ and CKD-EPI $(86,4 \pm 13,8$ vs $95,4 \pm 20,8)$ was observed between SSc patients with or without arterial hypertension.

None of 113 SSc patients, followed for a mean of $4.24 \pm 0.61$ years, have developed chronic renal insufficiency that progressed to dialysis.

We did notobserve significant differences renal Doppler and serological parameters between patients with renal complications and patients without renal complications.
Table 3. Median values and 95\% LC of Doppler indices of intrarenal stiffness, sCr, CKD-EPI, proteinuria 24h in SRC SSc patients at baseline (T0), during SRC (T1) and after median follow-up of 60 (9-60) months (T2). Pulsatile index (PI); resistive index (RI); systolic/diastolic ratio (S/D), serum creatinine (sCr); Chronic Kidney Disease Epidemiology Collaboration (CKD-EPI)

\begin{tabular}{lccccc}
\hline Variable & T0 & T1 & T2 & pT0-T1 & pT0-T2 \\
\hline PI & $1.67(1.56-1.69)$ & $1.54(1.48-1.84)$ & $1.70(1.60-1.75)$ & 0.81 & 0.048 \\
RI & $0.72(0.68-0.73)$ & $0.78(0.78-0.90)$ & $0.80(0.80-0.90)$ & 0.02 & 0.18 \\
S/D & $3.62(2.90-3.65)$ & $4.21(3.64-5.2)$ & $3.50(2.90-3.7)$ & 0.65 & 0.1 \\
sCr & $0.8(0.8-0.9)$ & $3.05(1.20-4.05)$ & $2.85(0.90-4)$ & 0.02 & 0.026 \\
CKD-EPI & $90(88-109)$ & $18(15-49)$ & $18(15-69)$ & 0.013 & 0.041 \\
Proteinuria 24h & $0.17(0.14-0.19)$ & $0.9(0.5-1.3)$ & $0.85(0.12-1.05)$ & 0.02 & 0.3 \\
\hline
\end{tabular}

Table 2. Mean values of Doppler indices of intrarenal stiffness, sCr, CKD-EPI, proteinuria $24 \mathrm{~h}$ and IMTin SSc patients at baseline (T0) and after follow-up of $4.1 \pm 0.9$ years (T1). Pulsatile index (PI); resistive index (RI); systolic/diastolic ratio (S/D), serum creatinine (sCr); Chronic Kidney Disease Epidemiology Collaboration (CKD-EPI)

\begin{tabular}{lccc}
\hline Variable & T0 & T1 & $\mathrm{p}$ \\
\hline PI & $1.43 \pm 0.28$ & $1.45 \pm 0.30$ & 0,78 \\
RI & $0.70 \pm 0.05$ & $0.70 \pm 0,07$ & 0,80 \\
S/D & $3.5 \pm 0.76$ & $3,5 \pm 0,80$ & 0,83 \\
SCr & $0.75 \pm 0.24$ & $0,78 \pm 0,26$ & 0,79 \\
CKD-EPI & $93.8 \pm 20$ & $90 \pm 25$ & 0,76 \\
Proteinuria 24h & $0.20 \pm 0.15$ & $0.19 \pm 0.18$ & 0,79 \\
IMT & $0.84 \pm 0.18$ & $0.83 \pm 0.21$ & 0,77 \\
\hline
\end{tabular}




\section{Kidney Blood Pressure Research}

Rosato et al.: Kidney and Systemic Sclerosis

Table 4. Mean values and SD of Doppler indices of intrarenal stiffness, sCrand CKD-EPI in three capillaroscopic groups. Pulsatile index (PI); resistive index (RI); systolic/diastolic ratio (S/D); serum creatinine (sCr); Chronic Kidney Disease Epidemiology Collaboration (CKD-EPI); Intimal media thickness (IMT)

\begin{tabular}{lcccc}
\hline Variable & Early & Active & Late & $\mathrm{p}$ \\
\hline PI & $1,32 \pm 0,26$ & $1,42 \pm 0,23$ & $1,52 \pm 0,32$ & 0,016 \\
RI & $0,67 \pm 0,05$ & $0,70 \pm 0,05$ & $0,72 \pm 0,06$ & $<0,0001$ \\
S/D & $3,12 \pm 0,69$ & $3,50 \pm 0,64$ & $3,75 \pm 0,81$ & 0,013 \\
sCr & $0,77 \pm 0,17$ & $0,73 \pm 0,23$ & $0,74 \pm 0,30$ & 0,30 \\
CKD-EPI & $93,3 \pm 18,9$ & $92,9 \pm 20$ & $95,1 \pm 21$ & 0,59 \\
IMT & $0,79 \pm 0,14$ & $0,85 \pm 0,19$ & $0,86 \pm 0,18$ & 0,22 \\
\hline
\end{tabular}

Table 5. Mean values and SD of Doppler indices of intrarenal stiffness, sCrand CKDEPI in SSc patients with and without digital ulcers (DUs). Pulsatile index (PI); resistive index (RI); systolic/diastolic ratio (S/D); serum creatinine (sCr); Chronic Kidney Disease Epidemiology Collaboration (CKD-

\begin{tabular}{lccc}
\hline Variable & SSc patients with DUs & SSc patients without DUs & $\mathrm{p}$ \\
\hline PI & $1,53 \pm 0,30$ & $1,32 \pm 0,21$ & $<0,0001$ \\
RI & $0,72 \pm 0,04$ & $0,68 \pm 0,04$ & $<0,0001$ \\
S/D & $3,77 \pm 0,82$ & $3,20 \pm 0,54$ & $<0,0001$ \\
SCr & $0,76 \pm 0,30$ & $0,74 \pm 0,15$ & 0,93 \\
CKD-EPI & $93,5 \pm 23,1$ & $94,1 \pm 16$ & 0,83 \\
IMT & $0,87 \pm 0,18$ & $0,81 \pm 0,17$ & 0,058 \\
\hline
\end{tabular}
EPI); Intimal media thickness (IMT)

\section{Discussion}

In our Scleroderma Unit, we have evaluated the clinical setting and long-term outcome of kidney disease secondary to SSc. According to studies published in the past 20 years, SRC in the United States has been reported in $10 \%$ of dcSSc patients and $2 \%$ of lcSSc patients [17]. Until a few years ago, SRC was the most severe complication and frequent cause of death reported in SSc [18]. Large studies have identified some factors that can be helpful in the evaluation of patients at high risk for SRC. Among these factors, some of them cannot be modified, such as autoantibody profile positive for anti-RNA polymerase III, arthritis, skin involvement and tendon friction rubs. The only modifiable factor is corticosteroid exposure $\geq 15 \mathrm{mg}$ per day in the preceding 6 months [19]. Although the reasons for this effect are not yet well understood, it is possible that high doses of corticosteroids can promote endothelial dysfunction and inhibit prostacyclin production, in turn increasing ACE activity [18]. In our study only six patients (4.9\%), five with diffuse SSc and one with limited SSc, developed SRC. Three patients had anti-topoisomerase antibody, two had anti RNA-polymerase III and only one had an anti-centromere antibody. Four of these six patients required hemodialysis and in two of them renal biopsy was performed because rapidly progressive oliguric renal failure since MPO-ANCA glomerulonephritis should be considered. One case of MPO-ANCA glomerulonephritis was diagnosed with renal biopsy while we have not registered associated APS nephropathy. In our cohort, one patient developed tubular acute necrosis, probably due to antibiotics (cephalosporin) toxicity. Several drugs hold the potential to cause renal damage, especially non-steroidal anti-inflammatory drugs able to induce tubulointerstitial nephritis. These manifestations are not related to SSc, and renal biopsy is required for accurate differential diagnosis. In the past, D-Penicillamine was administered to treat SSc, which was promoting renal complications like membranous glomerulopathy, proliferative crescentic glomerulonephritis and Goodpasture's Syndrome [4].

In SSc patients, renal function is normal despite the presence of increased intrarenal arterial stiffness. Renal function is preserved also in SSc patients with long disease duration. Age represents an independent risk factor for GFR decline [3]. Glomerular filtrate rate depends closely on the age and GFR remains constant on the mean values of $140 \mathrm{ml} /$ $\mathrm{min} / 1.73 \mathrm{~m}^{2}$ until the fourth decade then it declines by about $8 \mathrm{ml} / \mathrm{min} / 1.73 \mathrm{~m}^{2}$ per further decade [20]. Despite preserved renal function, in SSc patients Doppler indices of intrarenal arterial stiffness are higher than general population [3]. In our study, in SSc patients with arterial hypertension RI and SD were significantly higher than in patients without arterial 


\section{Kidney Blood Pressure Research}

hypertension. It is well known that hypertension is among major parameters that influence intrarenal resistance [16].

In this study we observed a negative correlation between Doppler indices of intrarenal arterial stiffness and estimated GFR evaluated by CKD-EPI. The Doppler indices of intrarenal arterial stiffness increased with progression of capillaroscopic damage and they are higher in SSc patients with DUs than in SSc patients without DUs. Subclinical and asymptomatic renal impairment is common in SSc and correlates with microvascular manifestations and prognosis. In previous studies, we have demonstrated that intrarenal arterial stiffness is increased significantly in SSc patients than healthy control and correlate with capillaroscopic damage [3]. Renal hemodynamic parameters negatively correlate with estimated and measured GFR and they are a marker of new DUs occurrence [21, 22]. In this study we did not observe significant differences of CKD-EPI between SSc patients with and without DUs. This difference compared to previous studies is due to the presence of different criteria of inclusion and exclusion (e.g. arterial hypertension). Many patients without DUs had arterial hypertension. Arterial hypertension is a risk factor for the reduction of GFR. In SSc patients, intrarenal arterial stiffness is considered an early marker in course of SRC [23] and cardiorenal syndrome [24]. Recently, Rosato et al. have demonstrated that RI are increased in SSc patients with anti-RNA polymerase III antibodies [25]. Thus, Doppler indices are reliable markers both in asymptomatic and in high risk renal vascular complications.

Based on these findings, we suggest that all patients with SSc should be carefully evaluated for symptomatic and asymptomatic renal involvement. Asymptomatic renal vasculopathy is frequently present but it does not seem to be associated with poorer outcome. Scleroderma Renal Crisis remains a severe manifestation of SSc despite treatment with high dose of angiotensin-converting enzyme inhibitor and dialysis.

\section{Conclusion}

In our cohort of SSc patients, renal function, evaluated by CKD-EPI, remained normal for 4.1 years despite the presence of increased intrarenal arterial stiffness. In addition, $4.9 \%$ of SSc patients developed SRC as main renal vascular complication. We can suggest that in SSc patients Doppler indices of intrarenal arterial stiffness should be performed in association with others markers of laboratory ( $\mathrm{sCr}$, urinalysis, estimated GFR, $24 \mathrm{~h}$ proteinuria) to evaluate vascular renal involvement. A renal biopsy should be strongly recommended to differentiate SRC from rapidly progressive glomerulonephritis such as MPO- ANCA positive associated or crescentic glomerulonephritis, because treatment regimens, patients' management as well as clinical outcomes are significantly different.

\section{Disclosure Statement}

The authors declare that the research was conducted in the absence of any commercial or financial relationships that could be construed as a potential conflict of interest. The authors are responsible for the content and writing of the paper. The manuscript is not under consideration for publication elsewhere.

\section{References}

1 Campbell PM, LeRoy EC: Pathogenesis of systemic sclerosis: a vascular hypothesis. Semin Arthritis Rheum 1975;4:351-368.

-2 Dowson C, Simpson N, Duffy L, O’Reilly S: Innate Immunity in Systemic Sclerosis. Curr Rheumatol Rep 2017;19:2. 


\section{Kidney \\ Blood Pressure Research}

Kidney Blood Press Res 2018;43:682-689
\begin{tabular}{l|l}
\hline DOI: 10.1159/000489740 & $\begin{array}{l}\text { c } 2018 \text { The Author(s). Published by S. Karger AG, Basel } \\
\text { www.karger.com/kbr }\end{array}$ \\
Published online: 16 May, 2018 &
\end{tabular}

Rosato et al.: Kidney and Systemic Sclerosis

-3 Rosato E, Gigante A, Barbano B, Cianci R, Molinaro I, Rossi C, Massa R, Amoroso A, Pisarri S, Salsano F: Intrarenal hemodynamic parameters correlate with glomerular filtration rate and digital microvascular damage in patients with systemic sclerosis. Semin Arthritis Rheum 2012;41:815-821.

4 Shanmugam VK, Steen VD: Renal manifestations in scleroderma: evidence for subclinical renal disease as a marker of vasculopathy. Int J Rheumatol 2010;2010:pii:538589.

5 Cannon PJ, Hassar M, Case DB, Casarella WJ, Sommers SC, LeRoy EC: The relationship of hypertension and renal failure in scleroderma (progressive systemic sclerosis) to structural and functional abnormalities of the renal cortical circulation. Medicine (Baltimore) 1974;53:1-46.

-6 Trostle DC, Bedetti CD, Steen VD, Al-Sabbagh MR, Zee B, Medsger TA Jr: Renal vascular histology and morphometry in systemic sclerosis. A case-control autopsy study. Arthritis Rheum 1988;31:393-400.

7 Siva B, McDonald SP, Hawley CM, Rosman JB, Brown FG, Wiggins KJ, Bannister KM, Campbell SB, Johnson DW: End-stage kidney disease due to scleroderma-outcomes in 127 consecutive ANZDATA registry cases. Nephrol Dial Transplant 2011;28:16.

-8 van den Hoogen F, Khanna D, Fransen J, Johnson SR, Baron M, Tyndall A, Matucci-Cerinic M, Naden RP, Medsger TA Jr, Carreira PE, Riemekasten G, Clements PJ, Denton CP, Distler O, Allanore Y, Furst DE, Gabrielli A, Mayes MD, van Laar JM, Seibold JR, et al.: 2013 classification criteria for systemic sclerosis: an American College of Rheumatology/European League against Rheumatism collaborative initiative. Arthritis Rheum 2013;65:2737-2747.

-9 LeRoy EC, Black C, Fleischmajer R, Jablonska S, Krieg T, Medsger TA Jr, Rowell N, Wollheim F: Scleroderma (systemic sclerosis): classification, subsets and pathogenesis. J Rheumatol 1988;15:202-2055.

10 Cutolo M, Sulli A, Secchi ME, Paolino S, Pizzorni C: Nailfold capillaroscopy is useful for the diagnosis and follow-up of autoimmune rheumatic diseases. A future tool for the analysis of microvascular heart involvement? Rheumatology 2006;45:43-46.

11 Clements P, Lachenbruch P, Siebold J, White B, Weiner S, Martin R, Weinstein A, Weisman M, Mayes M, Collier D et al.: Inter and intraobserver variability of total skin thickness score (modified Rodnan TSS) in systemic sclerosis. J Rheumatol 1995;22:1281-1285.

12 Valentini G, Della Rossa A, Bombardieri S, Bencivelli W, Silman AJ, D’Angelo S, Cerinic MM, Belch JF, Black CM, Bruhlmann P, Czirják L, De Luca A, Drosos AA, Ferri C, Gabrielli A, Giacomelli R, Hayem G, Inanc M, McHugh NJ, Nielsen H, et al.: European multicentre study to define disease activity criteria for systemic sclerosis. II. Identification of disease activity variables and development of preliminary activity indexes. Ann Rheum Dis 2001;60:592-598.

13 Medsger TA Jr, Silman AJ, Steen VD, Black CM, Akesson A, Bacon PA, Harris CA, Jablonska S, Jayson MI, Jimenez SA, Krieg T, Leroy EC, Maddison PJ, Russell ML, Schachter RK, Wollheim FA, Zacharaie H: A disease severity scale for systemic sclerosis: development and testing. J Rheumatol 1999;26:2159-2167.

14 Mancia G, Fagard R, Narkiewicz K, Redón J, Zanchetti A, Böhm M, Christiaens T, Cifkova R, De Backer G, Dominiczak A, Galderisi M, Grobbee DE, Jaarsma T, Kirchhof P, Kjeldsen SE, Laurent S, Manolis AJ, Nilsson PM, Ruilope LM, Schmieder RE, et al.: 2013 ESH/ESC Guidelines for the management of arterial hypertension. The Task Force for the management of arterial hypertension of the European Society of Hypertension (ESH) and of the European Society of Cardiology (ESC). J Hypertens 2013;31:1281-1357.

-15 Gigante A, Rosato E, Massa R, Rossi C, Barbano B, Cianci R, Molinaro I, Amoroso A, Salsano F: Evaluation of Chronic Kidney Disease Epidemiology Collaboration equation to estimate glomerular filtration rate in scleroderma patients. Rheumatology 2012;51:1426-1431.

16 Parolini C, Noce A, Staffolani E, Giarrizzo GF, Costanzi S, Splendiani G: Renal resistive index and long-term outcome in chronic nephropathies. Radiology 2009;252:888-896.

17 Steen VD, Medsger TA Jr, Osial TA Jr, Ziegler GL, Shapiro AP, Rodnan GP: Factors predicting development of renal involvement in progressive systemic sclerosis. Am J Med 1984;76:779-786.

18 Steen VD: Kidney involvement in systemic sclerosis. Presse Med 2014;43:305-314.

19 Shanmugam VK, Steen VD: Renal disease in scleroderma: an update on evaluation, risk stratification, pathogenesis and management. Curr Opin Rheumatol 2012;24:669-676.

20 Weinstein JR, Anderson S: The aging kidney: physiological changes. Adv Chronic Kidney Dis 2010;17:302307. 


\section{Kidney \\ Blood Pressure Research}

\section{Kidney Blood Press Res 2018;43:682-689}

\begin{tabular}{l|l}
\hline DOI: $10.1159 / 000489740$ & (C) 2018 The Author(s). Published by S. Karger AG, Basel
\end{tabular}

Published online: 16 May, 2018 www.karger.com/kbr

Rosato et al.: Kidney and Systemic Sclerosis

21 Gigante A, Barbano B, Granata G, Quarta S, Amoroso A, Salsano F, Cianci R, Rosato E: Evaluation of estimated glomerular filtration rate and clinical variables in systemic sclerosis patients. Clin Nephrol 2016;85:326331.

22 Rosato E, Barbano B, Gigante A, Molinaro I, Quarta S, Pisarri S, Amoroso A, Cianci R, Salsano F: Increased intrarenal arterial stiffness may predict the occurrence of new digital ulcers in systemic sclerosis. Arthritis Care Res 2014;66:1380-1385.

-23 Rosato E, Gigante A, Barbano B, Molinaro I, Cianci R, Salsano F: Doppler indices of intrarenal arterial stiffness are useful in monitoring scleroderma renal crisis. Scand J Rheumatol 2013;42:80-81.

-24 Gigante A, Barilaro G, Barbano B, Romaniello A, Di Mario F, Quarta S, Gasperini ML, Di Lazzaro Giraldi G, Laviano A, Amoroso A, Cianci R, Rosato E: Left ventricular mass and intrarenal arterial stiffness as early diagnostic markers in cardiorenal syndrome type 5 due to systemic sclerosis. Cardiorenal Med 2016;6:135142.

-25 Rosato E, Navarini L, Gigante A, Cianci R, Margiotta D, Barbano B, Afeltra A: Intrarenal arterial stiffness is increased in systemic sclerosis patients with anti-ribonucleic acid polymerase III antibodies. Rheumatology 2017;56:1039-1041. 